\title{
SECADO DE ASERRIN EN LECHO FLUIDIZADO
}

Rogelio Moreno M. (*)

\section{RESUMEN}

En este trabajo se dan a conocer los resultados experimentales obtenidos para el secado de aserrin en lecho fluidizado.

Para tal efecto se construyó un banco de ensayos de transferencia de calor y materia con el fin de obtener las curvas y tiempos de secado del material particulado.

Adicionalmente, se proporcionan algunos indicadores sobre la capacidad de producción y de consumo de energía.

El empleo de la técnica será ventajoso en la medida que se disponga de energía calórica gratuita disponible en productos de combustión, por ejemplo.

Palabras claves: Aserrín. Secado.Fluidización.

\section{ABSTRACT}

This paper provides the experimental results obtained for sawdust drying in fluidized bed.

An experimental fluidized bed both for heat and mass tranfer was built with this purpose. so as to get the curves and times of drying of the granular material.

In addition, some indicators are provided to show the production capacity and energy consumption.

The use of this technique will be advantageous if free caloric energy is available in, for example, combustion products.

Keywords: Sawdust. Drying. Fluidization. 


\section{INTRODUCCION}

Una de las alternativas que se vislumbran como factibles para el uso racional del aserrín es la combustión con el fin de generar energía y al mismo tiempo evitar la creciente acumulación de este desecho forestal (Wagemann, 1984 a). Sin embargo, debido a los altos contenidos del humedad que posee, especialmente en la zona sur del país donde se da en mayores cantidades, se produce una utilización ineficiente de su poder calorifico y se requiere de mayores volúmenes de cámaras de combustión para realizar el proceso.

En Chile, hay pocos estudios tendientes a encontrar una solución técnica y económicamente atractiva para el secado de aserrín y así quemarlo en mejores condiciones.

Por otro lado, el empleo de la técnica de la fluidización en el secado de productos, ha cobrado gran importancia en los últimos años, debido a las características del contacto sólido-gas que origina altas velocidades de transporte de calor y materia entre ambos medios y, por tanto, reduce notablemente los tiempos de proceso.

La fluidización consiste en mantener en suspensión o flotación a los sólidos que en este caso particular se desean secar, por medio de una corriente de aire ascendente tal como se muestra en la Figura №1. Así, el aire, además de tener adecuadas condiciones psicrométricas (temperatura y húmedad) debe poseer una velocidad suficientemente alta para producir una fuerza de arrastre (Fa) sobre los sólidos, de igual magnitud al peso efectivo de ellos (peso menos empuje). En Apéndice $\mathrm{N}^{\circ} 1$ se indica la nomenclatura utilizada.

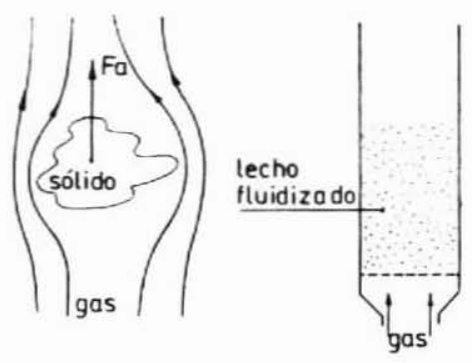

Figura N ${ }^{\circ} 1$. ESQUEMA BASICO dE LA FLUIDIZACION. 
Muchos son los parámetros que intervienen en la calidad y velocidad del proceso, algunos relacionados con el mecanismo interno de circulación de agua en los sólidos y otros relacionados con las condiciones exteriores tales como temperatura, humedad y velocidad del gas. También influye el tamaño y forma de las partículas, la geometría del lecho, y la distribución del gas, entre otros factores.

No obstante el desarrollo de la técnica que ha dado origen a una gran cantidad de información teórico-experimental relacionada con el tema (Vanecek et al., 1966 a; Kunii et al., 1969; Davidson et al., 1985, entre otros), en el caso particular de sólidos tan irregulares como el aserrín no parece confiable basar el diseño o proyecto de unidades de secado en ecuaciones o resultados obtenidos para sólidos de otras características físicas y geométricas y bajo otras condiciones de operación.

Así lo demuestran los resultados obtenidos por el autor en estudios experimentales relacionados con caracterización de sólidos y comportamiento fluidodinámico de un lecho fluidizado de aserrín (Moreno, 1989; Moreno, 1990). Se detectaron importantes desviaciones entre los valores experimentales y los que predicen correlaciones entregadas por la literatura para parámetros relevantes, como velocidad mínima de fluidización, velocidad de arrastre y pérdidas de carga. Las distorsiones se atribuyen principalmente a la irregularidad de los sólidos, en cuanto a granulometría y forma de partículas, y a la tendencia a la aglomeración que tienen las partículas, especialmente las de pequeño tamaño y gran contenido de húmedad.

En base a lo anteriormente expuesto, el objetivo de este trabajo consiste en obtener experimentalmente resultados relativos al secado de aserrin en lecho fluidizado en un equipo de laboratorio.

Aunque el trabajo no representa una respuesta completa al problema, pues se deben realizar estudios a escala mayor, se obtienen bajos tiempos de secado debido a las altas tasas de transferencia de calor y materia que se producen entre el aserrin y el aire.

\section{MATERIAL Y METODO}

El desarrollo del trabajo se efectuó en un banco de ensayos de transferencia de calor y materia al cual se le introdujeron las muestras de aserrín de Pino Insigne previamente caracterizadas. Estas provenian del proceso de aserrado de madera en la sierra múltiple del Aserradero Vista Alegre de la Universidad Austral de Chile. 
Las condiciones de operación del equipo se establecieron en base a trabajos previos del autor mencionados anteriormente. Específicamente, para el diseño del calefactor eléctrico se consideraron velocidades de operación entre 1 y $4 \mathrm{~m} / \mathrm{s}$ y temperaturas normalmente empleadas en el proceso de secado, es decir, 50 a $110^{\circ} \mathrm{C}$. Con estos antecedentes se construyó el calentador de aire con una capacidad variable entre 0 y $2 \mathrm{Kw}$.

Por otro lado, debido a la diversidad de tamaño de las particulas de aserrín, se debió realizar una caracterización de ellas, ya que esta variable juega un papel importante en el dimensionamiento de unidades de lecho fluidizado.

Uno de los procedimientos seguidos para efectuar estudios granulométricos de materiales particulados polidispersos, es el empleo de tamices (Vanecek et al., 1966 b). El tamaño de una fracción $x_{i}$ de particulas retenidas entre dos tamices de aberturas $d_{i}$ y $d_{2}$, se calculó como:

$$
\mathrm{dp}_{\mathrm{i}}=\frac{\mathrm{d}_{\mathrm{i}}+\mathrm{d}_{2}}{2}
$$

El diámetro medio de una muestra se obtuvo mediante la expresión que proporciona el valor medio ponderado con el peso.

$$
d p=\frac{\Sigma m_{i} g d p_{i}}{\Sigma m_{i} g}=\Sigma x_{i} d p_{i}
$$

Se efectuaron ensayos con tamices normalizados ASTM y muestras de aserrín previamente caracterizadas y con húmedad higroscópica. Estas muestras correspondian a una parte del aserrín posteriormente colocado en el lecho. En la Figura № 2 se muestra la distribución de tamaño de dos muestras de aserrín y en la Figura $\mathrm{N}^{\circ} \mathrm{3}$ el equipo experimental.

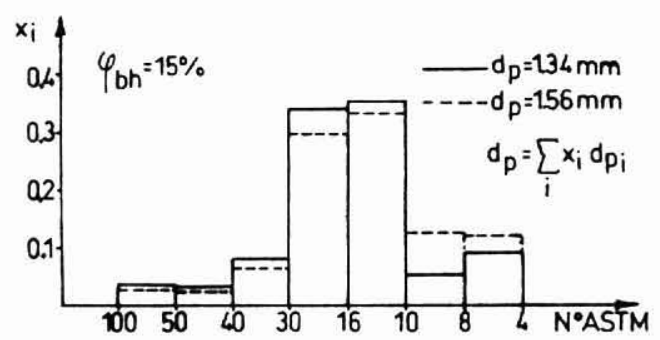

Figura $N^{\circ} 2$ Distribucion de taMAÑo de PARTiCULAS. 


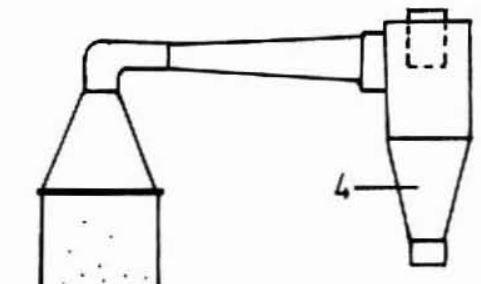

1. Lecho de particulas; $D=0.1 \mathrm{~m}, H=0.6 \mathrm{~m}$

2. Distribuidor de aire; malla mesh 100

3. Calefactor eléctrico: cap $=0-2 \mathrm{Kw}$

4. Ciclón recolector de finos

5. Anemómetro de aletas; resol.: $0.1 \mathrm{~m} / \mathrm{s}$

6. Válvula alimentadora de aire

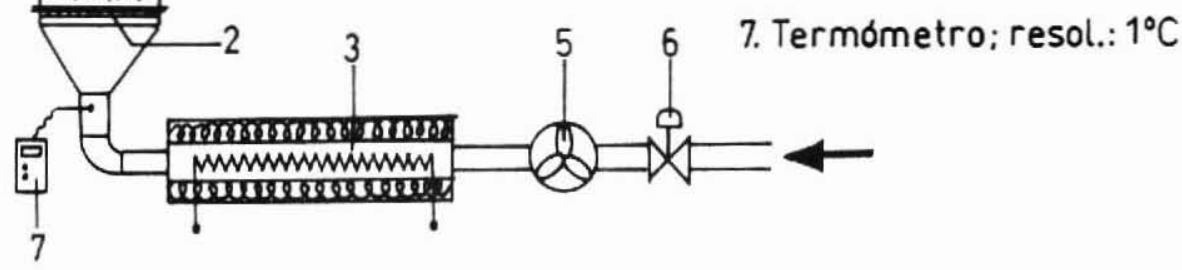

Figura $N^{\circ} 3$ esquema general del EQUiPO EXPERIMENTAL.

Los parámetros seleccionados para los ensayos fueron temperatura del fluidizante (aire) y tamaño de partículas. En cuanto a la velocidad de operación del lecho (velocidad superficial del aire), se puede decir que producto de la aglomeración de sólidos que se origina para altos contenidos de húmedad de ellos, en el inicio del proceso de secado se realiza con el lecho en reposo, no siendo posible la fluidización aún empleando altas velocidades. A medida que el proceso avanza y dismimuye la humedad del aserrín, la calidad de la fluidización crece, pasando a una fluidización tipo pistón y fluidización canalizada para finalizar con una fluidización prácticamente completa.

Dicho fenómeno hizo necesario el empleo de altas velocidades superticiales al inicio del proceso, para luego ir disminuyendo gradualmente y así evitar, en cierto grado, el arrastre de sólidos más finos que van perdiendo humedad. A medida que transcurría el tiempo de secado se extrajeron pequeñas muestras de sólidos del lecho y se llevaron al laboratorio para la determinación de su humedad a través de secado y diferencia de pesadas. Se construyeron así las curvas de secado para diferentes valores de temperaturas del aire y varios tamaños de partículas. 


\section{RESULTADOS}

Los resultados se muestran en las Figuras $\mathrm{N}^{\circ} 4$ y $\mathrm{N}^{\circ} 5$; en el primer caso se trata de aserrín polidisperso, con un diámetro medio ponderado de partículas igual a $1.34 \mathrm{~mm}$ y temperaturas variable entre 60 y $110^{\circ} \mathrm{C}$. En el segundo caso se trabajó con una temperatura del aire constante y con aserrín de tamaños seleccionados por tamizado. Se verifica que el empleo de altas temperaturas acelera el proceso y que el secado de aserrín de partículas pequeñas es más rápido debido a las altas superficies de intercambio de calor y materia que presentan los sólidos en relación al fluidizante.

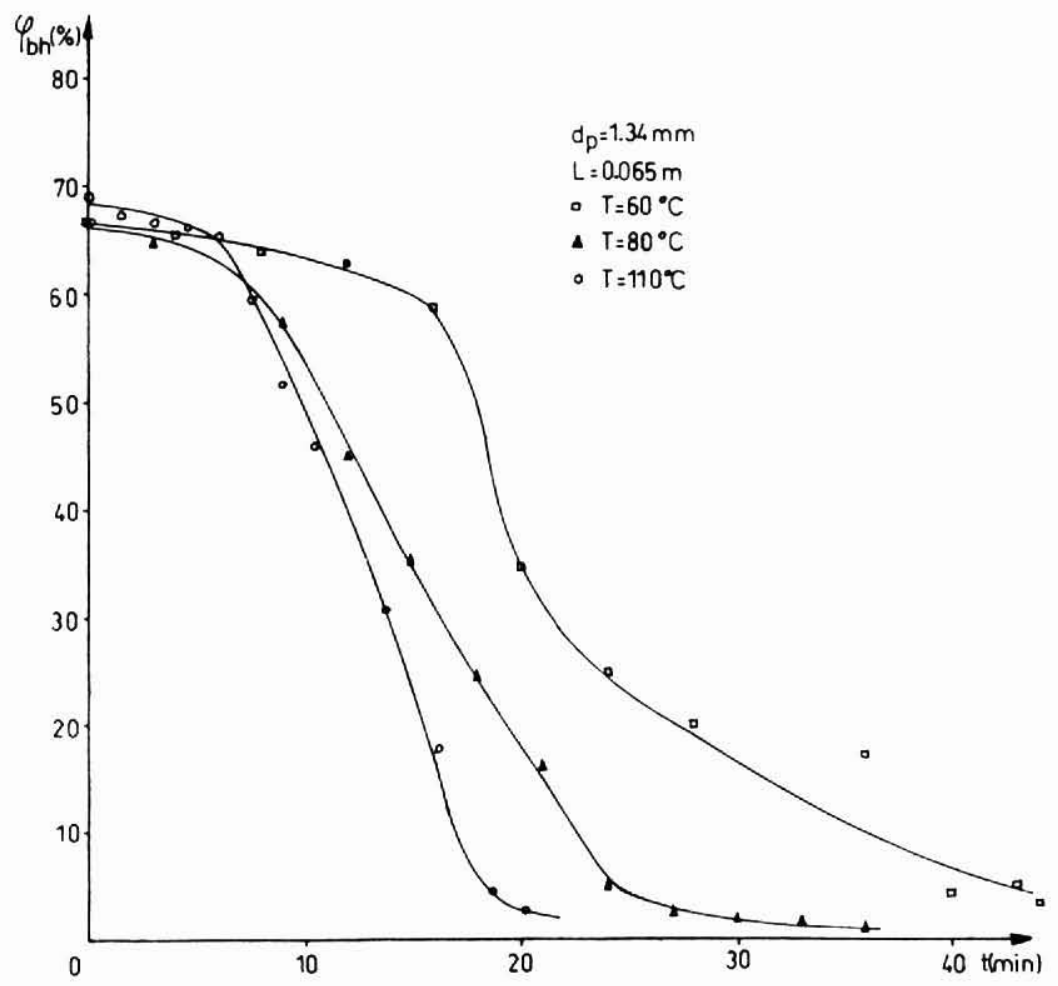

Figura N ${ }^{\circ} 4$ CURVAS de SECAdO PARA DIFERENTES TEMPERATURAS DEL AIRE. 


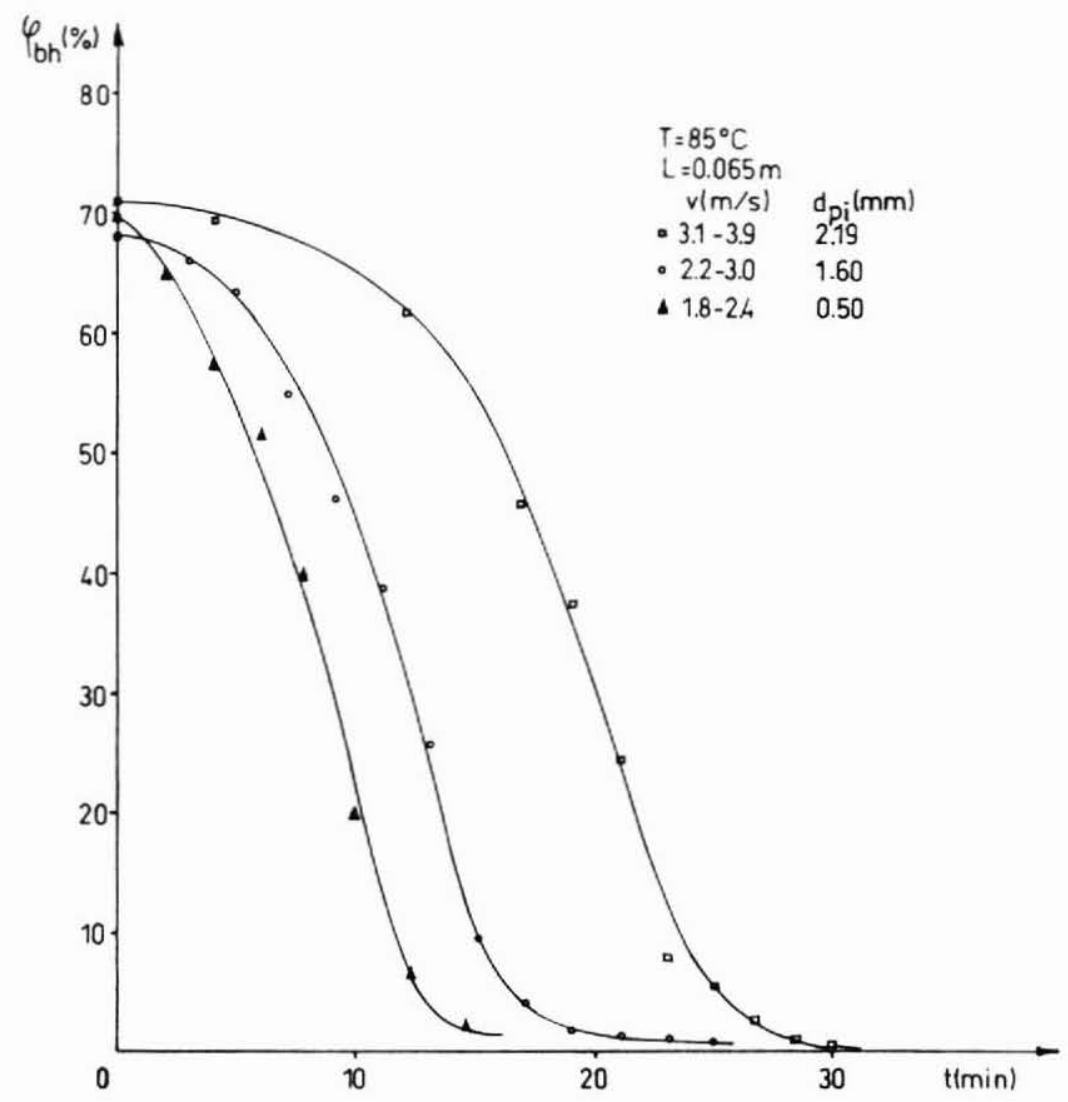

Figura N $N^{\circ} 5$ CURVAS dE SECAdO PARA DIFERENTES TAMAÑOS DE PARTICULAS.

\section{DISCUSION}

Es importante señalar que las diferencias entre las curvas de la Figura $\mathrm{N}^{\circ} 5$ están atenuadas en cierto grado ya que para tamaños grandes de particulas, aunque el tiempo requerido es mayor, este efecto es compensado parcialmente por el hecho de tener que emplear mayores velocidades y por una menor tendencia a la aglomeración. 
Los breves tiempos requeridos para el secado desde humedades del orden de $65-75 \%$ (base húmeda) hasta la humedad de equilibrio de $10-16 \%$, le otorgan una capacidad de producción al equipo de $32 \mathrm{Kg} / \mathrm{h}$ de producto seco polidisperso por $\mathrm{m}^{2}$ de sección transversal del lecho, empleando $80^{\circ} \mathrm{C}$ de temperatura del aire y una altura de lecho de $0,1 \mathrm{~m}$. Este resultado es muy superior al obtenido por medio de un secador eólico solar cuya capacidad de producción fue de $2 \mathrm{Kg} / \mathrm{m}^{2}$ dia (Wagemann, 1984 b). Esto se atribuye a que en el segundo caso el aserrín se secó en lecho fijo y sólo eventualmente, cuando se presentaban grandes vientos se producía una tendencia a la fluidización y a la separación de los sólidos en el secador. En el primer caso la fluidización se consiguió mecánicamente acelerando considerablemente el proceso de extracción de humedad hasta conseguir la evaporación de $72 \mathrm{Kg} / \mathrm{h}$ de agua por $\mathrm{m}^{2}$ de lecho.

En cuanto al consumo de energía del proceso, por un lado se tiene la energía calórica necesaria para calentar el aire y, por otro, la energía mecánica requerida para producir el fenómeno de la fluidización por medio de un ventilador.

En el primer caso los consumos fueron elevados, alrededor de $2.500 \mathrm{Kcal} / \mathrm{Kg}$ de agua evaporada, consiguiendose un rendimiento térmico de secado de $25 \%$. (parametro que no fue evaluado en el trabajo de Wagemann). Por tanto, desde el punto de vista de la rentabilidad, el empleo de la fluidización en el secado será justificable si se dispone de energía calórica, por ejemplo en gases de combustión de algún otro proceso industrial.

En todo caso, cualitativamente se espera que en unidades de gran tamaño puedan emplearse mayores alturas de lecho, ya que no se tendrá el efecto de pared que se tuvo en el caso aqui presentado, por tratarse de un equipo de laboratorio de pequeño diámetro. Este efecto de pared produce una mayor tendencia a la aglomeración de los sólidos. El empleo de mayores alturas de lecho obviamente aumentará la capacidad de producción, disminuirá el consumo específico de energía y aumentará la eficiencia de la unidad pues el aire se evacuará al ambiente a menores temperaturas. En términos cuantitativos no hay abundante información al respecto, solo se sabe que hay algunas unidades de secado de aserrín operando con capacidades del orden de $150-200 \mathrm{Kg} / \mathrm{h}$ de agua evaporada por $\mathrm{m}^{2}$ de lecho y consumos específicos de calor de 850 - $1250 \mathrm{Kcal} / \mathrm{Kg}$ de agua evaporada (Vanecek et., al $1966 \mathrm{c}$ ).

En relación al consumo de energía del ventilador, aún no hay claridad absoluta, pues sólo se conocen los valores de pérdidas de carga correspondientes al lecho (Moreno, 1989), quedando pendiente aún la evaluación del sistema (intercambiador de calor, ductos, etc). Esto último dependerá en gran medida del tamaño de la unidad.

Los resultados obtenidos en este estudio también pueden ser de interés en el 
proceso de fabricación de briquetas de aserrín, donde se requiere que el material particulado tenga una humedad del orden de 15 a $20 \%$.

\section{CONCLUSIONES}

Aunque la fluidización del aserrín es posible de obtener, hay una gran tendencia a la aglomeración de partículas cuando su contenido de húmedad supera el 45 $-50 \%$. Esto produce fenómenos de flujo pistón y canalización, los que se pueden atenuar en algún grado si se emplean bajas alturas de lecho (inferiores a $0.1 \mathrm{~m}$ ) en equipos de pequeño diámetro $(0.1 \mathrm{~m})$.

A través de la fluidización del aserrín se obtienen tiempos de secado considerablemente inferiores, en relación a los conseguidos con secadores que emplean energía natural (eólico-solar). Se requieren tiempos inferiores a 1 hora para reducir la húmedad de los sólidos desde $65-75 \%$ hasta $10-15 \%$.

La capacidad de producción del proceso, en su fase experimental, es del orden de $32 \mathrm{Kg} / \mathrm{h}$ de producto seco, por $\mathrm{m}^{2}$ de sección transversal de lecho, cuando se emplean $80^{\circ} \mathrm{C}$ de tempertura del aire, alturas de lecho de $0.1 \mathrm{~m}$ y partículas polidispersas de aserrín con un promedio ponderado de $1.34 \mathrm{~mm}$.

El consumo de energía calórica es elevado por lo que el proceso será rentable si se dispone de calor residual de algún otro proceso productivo industrial.

Se deben realizar estudios a gran escala para obtener resultados más completos sobre el tema. Una idea también interesante, debido a los bajos tiempos de secado del material particulado, consiste en intentar quemar aserrín húmedo en un lecho fluidizado pues debería ser más atractivo que quemarlo en calderas convencionales donde el combustible se quema en reposo y por tanto en forma lenta.

\section{RECONOCIMIENTOS}

Este trabajo se realizó gracias al apoyo brindado por la Dirección de Investigación y Desarrollo de la Universidad Austral de Chile al Proyecto de Investigación S-89-31. 


\section{REFERENCIAS BIBLIOGRAFICAS}

Davison, J.F. et al., 1985. Fluidization, Academic Press, Inc., London.

Kunii, D. et al., 1969. Fluidization Engineering, John Wiley \& Sons, Inc., New-York.

Moreno, R., 1989. Comportamiento hidrodinámico de un lecho fluidizado de aserrín. Anales X Congreso Brasileiro de Engenharia Mecánica, 1: 359 - 362, Rio de Janeiro.

Moreno, R., 1990. Fluidización de aserrín: Experimentación y estudio de correlaciones. Anales IV Congreso Nacional de Ingeniería Mecánica, 332 - 343, Santiago.

Vanecek, V et al., 1966 a. Fluidized Bed Drying, Leonard Hill, London.

Vanecek, V. et al., 1966 b. op. cit., pp. 20.

Vanecek, V. et al., 1966 c. op. cit., pp. 156 - 157

Wagemann G., 1984 a. Problemas en la utilización de desechos de madera en Chile, Anales Segunda Semana de la Energía, 1:59 - 69, Temuco.

Wagemann, G., 1984 b. Problemas de Secado del Aserrin, Anales Segunda Semana de la Energia, 1: 71-82, Temuco. 


\section{APENDICE $N^{\circ} 1$}

dp : Diametro medio de partículas ponderado con el peso ( $\mathrm{mm})$.

$\mathrm{dp}_{\mathrm{i}} \quad$ : Diametro promedio de partículas entre dos tamices consecutivos (mm).

$d_{1}, d_{2}$ : Tamaños de dos aberturas consecutivas de tamices (mm)

D : Diámetro de la columna del lecho $(\mathrm{m})$

$\mathrm{Fa} \quad$ : Fuerza de arrastre $(\mathrm{Kg})$

$\mathrm{H} \quad$ : Altura de la columna del lecho $(\mathrm{mm})$

L : Altura del lecho de partículas (m)

t : Tiempo de secado (min)

$\mathrm{T} \quad$ : Tempertura de entrada del aire al lecho $\left({ }^{\circ} \mathrm{C}\right)$

$\checkmark \quad$ : Velocidad superficial del aire $(\mathrm{m} / \mathrm{s})$

$\mathrm{x}_{\mathrm{i}} \quad$ : Fracción de partículas de tamaño $\mathrm{dp}_{\mathrm{i}}$

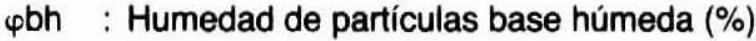

